\title{
Florística de uma floresta estacional no Planalto da Borborema, nordeste do Brasil
}

\author{
Floristic of seasonal forest in the Borborema Plateau, northeastern Brazil
}

\author{
Ladivania Medeiros do Nascimento ${ }^{1,4}$, Maria Jesus Nogueira Rodal ${ }^{1}$ \& Alexandre Gomes da Silva ${ }^{3}$
}

\begin{abstract}
Resumo
O conhecimento disponível sobre a flora das florestas montanas (Brejo de Altitude) do semiárido de Pernambuco aponta duas situações: uma com maior semelhança à das florestas mais próximas à costa atlântica; e outra mais similar às florestas mais secas do sertão nordestino. Este trabalho teve como objetivo investigar a composição de fragmentos de floresta montana no município de Brejo da Madre de Deus, Pernambuco, situados numa área geográfica de transição entre aqueles tipos florestais. Os resultados foram comparados com cinco levantamentos florísticos realizados em florestas pernambucanas que utilizaram metodologia similar de amostragem. Foram identificadas 293 espécies, distribuídas em 185 gêneros e 71 famílias. As espécies arbóreas e arbustivas/subarbustivas se destacaram em riqueza total de espécies coletadas $(50 \%$ e $24 \%$, respectivamente), indicando similaridade florística com as florestas montanas mais próximas à costa atlântica. Os outros hábitos não apresentaram padrão claro de similaridade florística.
\end{abstract}

Palavras-chave: Brejo de Altitude, composição florística, floresta montana, fragmento.

\begin{abstract}
The information available on the flora of upland forests of Pernambuco's semi-arid region ("brejo de altitude") indicates two situations: one flora more similar with forests closer to the Atlantic coast; and another more similar to the drier forests of northeastern inlands. This study aimed to investigate the floristic composition of upland forest fragments in the municipality of Brejo da Madre de Deus, Pernambuco, located in a geographical transition area between those forests. The results were compared with five floristic surveys carried out in Pernambuco forests that used similar sampling methodologies. Two-hundred ninety-three species were identified, distributed among 185 genera and 71 families. Arboreal and shrubby/subshrub species stood out richness of the total species collected (50\% and $24 \%$, respectively), which indicates floristic similarity with the highland forests closer to the Atlantic coast. The other habits did not present clear floristic similarity patterns.
\end{abstract}

Key words: Brejo de Altitude, floristic composition, fragments, montane forest.

\section{Introdução}

O conhecimento atual da flora de angiospermas das florestas do leste da América do Sul mostra que, quando as análises florísticas são restritas a setores da Floresta Atlântica, como os do nordeste e do sudeste brasileiro, o padrão das espécies parece ser primariamente relacionado com o clima, diferenciando as florestas ombrófilas das estacionais (Oliveira-Filho et al. 2006).
No nordeste brasileiro, as florestas estacionais ocorrem nos limites entre a Floresta Atlântica a leste e ao longo de toda a zona de contato entre as Caatingas e os Cerrados a oeste (IBGE 1993), acompanhando a isoieta de $1.000 \mathrm{~mm}$ de precipitação anual (Andrade Lima 1981). Sabe-se que sua composição varia em função de vários fatores, especialmente a distância do mar; e que àquelas presentes em áreas de maior altitude dentro

Este artigo possui material adicional em sua versão eletrônica.

${ }^{1}$ Programa de Pós-Graduação em Botânica, Universidade Federal Rural de Pernambuco, R. D. Manoel de Medeiros s/n, 52171-900, Recife, PE, Brasil.

${ }^{2}$ Universidade Federal Rural de Pernambuco, Depto. Biologia, R. D. Manoel de Medeiros s/n, 52171-900, Recife, PE, Brasil.

${ }^{3}$ Programa de Pós-Graduação em Botânica da Escola Nacional de Botânica Tropical do Rio de Janeiro, Instituto de Pesquisas Jardim Botânico do Rio de Janeiro, 22460-000, RJ, Brasil.

${ }^{4}$ Autor para correspondência: ladivania@hotmail.com 
do semiárido, os chamados Brejos de Altitude, notabilizam-se por estarem circundadas por Caatinga (Rodal et al. 2008).

Os Brejos de Altitude ocupam as áreas mais elevadas do planalto da Borborema (acima de 600 $\mathrm{m})$, sendo classificados como florestas montanas por Veloso et al. (1991). Seu surgimento em pleno semiárido resulta de uma condição climática especial em função da altitude e do relevo, que criam uma situação particular onde as massas de ar depositam umidade na encosta de grandes maciços e planaltos voltados para a direção do vento (Andrade-Lima 1981). Essa condição climática especial cria a chamada "precipitação oculta" que propicia uma intensa condensação noturna, especialmente nos meses mais frios, levando ao desenvolvimento de uma vegetação florestal em pleno semiárido (Andrade \& Lins 1965), a qual desempenha relevante função de proteção de nascentes de rios e de lençóis freáticos (AndradeLima 1966).

A conservação dessas florestas montanas é crítica, uma vez que no inicio dos anos 70 Vasconcelos-Sobrinho (1971) identificou a existência de $8.569 \mathrm{~km}^{2}$ de remanescentes dessas florestas nos estados do Ceará, Rio Grande do Norte, Paraíba e Pernambuco. Trinta anos mais tarde, Tabarelli \& Santos (2004) afirmaram que na mesma região restam apenas $2.626 \mathrm{~km}^{2} \mathrm{e}$ pouco mais de $30 \mathrm{~km}^{2}(0,16 \%)$ são protegidos por unidades de conservação.

O conhecimento botânico dessas florestas em Pernambuco está disponível em uma série de estudos pontuais em áreas mais interioranas situadas no sertão (Sales et al. 1998; Rodal \& Nascimento 2006; Nascimento \& Rodal 2008) e de levantamentos em áreas mais próximas da costa atlântica (Rodal et al. 2005a; Ferraz \& Rodal 2006; Ferraz \& Rodal 2008), havendo uma lacuna de informação sobre as florestas situadas na zona de transição entre o litoral e o sertão do Estado.

A síntese dos trabalhos acima citados aponta inicialmente para dois padrões florísticos: o das florestas montanas mais próximas à costa atlântica, onde a flora parece ser um prolongamento da floresta úmida costeira; e o das mais interioranas, localizadas no sertão do Estado, onde há uma flora diferenciada mais similar às florestas mais secas do sertão nordestino. Este trabalho investiga a flora de angiospermas numa floresta de transição em Pernambuco, visando melhor entender o padrão florístico destas florestas ameaçadas.

\section{Material e Métodos}

Área de estudo

A Serra do Bituri situa-se no município de Brejo da Madre de Deus, Pernambuco, no Planalto da Borborema, em uma área de transição entre o litoral e o sertão $\left(8^{\circ} 12^{\prime} 05^{\prime \prime}-8^{\circ} 12^{\prime} 41,5^{\prime \prime} \mathrm{S}\right.$ e $36^{\circ} 23^{\prime} 21^{\prime \prime}-36^{\circ} 23^{\prime} 73^{\prime \prime} \mathrm{W}$ ) (Fig. 1). Essa área representa apresenta um conjunto de fragmentos de floresta estacional semidecídua montana (Veloso et al. 1991), com altitude entre 900 e $1.100 \mathrm{~m}$. A temperatura média anual é de $22,2^{\circ} \mathrm{C}$, com médias máximas mais elevadas entre dezembro e janeiro de $23,5^{\circ} \mathrm{C}$; e mínimas entre julho, agosto e setembro de $16,5^{\circ} \mathrm{C}$. A precipitação média anual é de $948 \mathrm{~mm}$, com período de chuvas se estendendo de março a julho e período seco $\left(<100 \mathrm{~mm}\right.$ mês $\left.^{-1}\right)$ de seis a oito meses (Silva et al. 2009), o que caracteriza o clima como tropical úmido-seco, com longa estação seca (Richards 1996).

Os fragmentos estudados estão em propriedade particular, em geral rodeados por áreas com cultivo extensivo do café, banana, cenoura e beterraba, resultando em uma paisagem extremamente fragmentada. No entanto, como alguns fragmentos estão em bom estado de conservação, foram transformados em Reserva Particular do Patrimônio Natural (RPPN), a exemplo da RPPN Fazenda Bituri.

\section{Procedimento amostral e análise}

estatística

As coletas foram realizadas mensalmente por um período de dois anos (1999-2002) através do método de caminhamento (Filgueiras et al. 1994), seguindo as técnicas usuais de coleta para plantas (Mori et al. 1989). As exsicatas foram incorporadas ao herbário Professor VasconcelosSobrinho (PEUFR) do Departamento de Biologia da UFRPE, onde foram devidamente catalogadas. A identificação das espécies foi realizada por comparação com exsicatas depositadas nos herbários PEUFR, IPA e por especialistas de diferentes herbários do Brasil e do exterior. As espécies de Myrtaceae foram comparadas às identificações do material coletado em Brejo da Madre de Deus e outras florestas montanas de Pernambuco durante a execução do projeto "Composição florística e diversidade nos Brejos de Altitude em Pernambuco", colaboração entre o Departamento de Biologia da Universidade Federal Rural de Pernambuco e o Royal Botanic Gardens, Kew. 


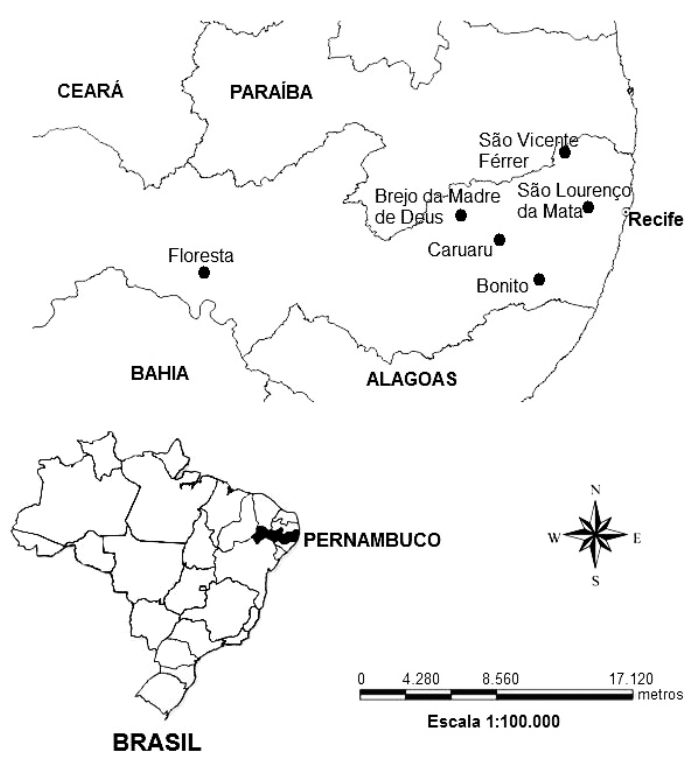

Figura 1 - Localização do município de Brejo da Madre de Deus (área de estudo) e outros utilizados para comparação florística (Caruaru, Floresta, Bonito, São Lourenço da Mata, São Vicente Férrer), Pernambuco, Brasil.

Figure 1 - Localization of Madre de Deus municipality (study area) and other floristic surveys used to floristic analysis ( $\mathrm{Ca}$ ruaru, Floresta, Bonito, São Lourenço da Mata, São Vicente Ferrer), Pernambuco, Brazil.

Foi elaborada uma lista das espécies ordenadas por família, com informações acerca do hábito (Font-Quer 2000), nome e número do coletor e determinador. A classificação de famílias adotou a proposta do APG III (2009) e a grafia dos autores dos nomes científicos seguiu o site do IPNI (2010).

A partir dessa lista, foi elaborada uma tabela com as famílias de maior riqueza de espécies da área e comparado com os de cinco levantamentos qualitativos em áreas de floresta montana que utilizaram o mesmo método de coleta (Caruaru (Rodal \& Sales 2008); Floresta (Rodal \& Nascimento 2002); São Vicente Férrer (Ferraz \& Rodal 2008); Bonito (Rodal et al. 2005b) e um levantamento em uma área de floresta de terras baixas em São Lourenço da Mata (Rodal et al. 2005a).

Com base nesses mesmos levantamentos, foram montadas três matrizes de dados binários, apenas com taxa identificados no nível específico, uma com as espécies arbóreas, uma com as arbustivas/subarbustivas e uma com as herbáceas/ trepadeiras. A partir dessas matrizes foi realizada uma análise de agrupamento, utilizando o índice de Jaccard e o método de ligação da média do grupo (UPGMA), processada pelo programa PC-ORD for Windows versão 4.0 (McCune \& Mefford 1999), cujos resultados foram plotados em três dendrogramas.

\section{Resultados e Discussão}

Foram registradas 290 espécies, distribuídas em 185 gêneros e 71 famílias (Apêndice 1). A identificação no nível de espécie foi de $89 \%$. Destaque para Fabaceae (30 espécies), Myrtaceae (24 spp.), Asteraceae (23 spp.), Solanaceae e Rubiaceae (15 spp. cada), Orchidaceae e Melastomataceae (13 spp. cada) e Euphorbiaceae (12 spp.), totalizando $51 \%$ das espécies amostradas. Apesar das oito famílias de maior riqueza específica nos fragmentos da Serra do Bituri serem praticamente as mesmas de outras florestas do litoral e interior de Pernambuco (Tab. 1 ), nota-se que existem variações na composição de espécies.

Árvores - Trata-se do hábito com maior riqueza de espécies (127), cerca de 50\% do total das plantas coletadas. Na área de estudo e em dois levantamentos listados na Tabela 2 (São Vicente Férrer e São Lourenço da Mata) também houve maior proporção de espécies arbóreas. Segundo Richards (1996) a riqueza de espécies arbóreas é uma das características mais marcantes das diferentes tipologias florestais tropicais.

Fabaceae (23 espécies) e Myrtaceae (17) são os taxa com maior riqueza de espécies arbóreas do dossel e sub-dossel da floresta, semelhante ao registrado nas florestas consideradas na Tabela 2 e em levantamentos realizados em florestas do domínio atlântico de Pernambuco (Guedes 1998; Sales et al. 1998; Moura \& Sampaio 2001; Siqueira et al. 2001), de outros Estados do Nordeste (Lourenço \& Barbosa 2003; Cestaro \& Soares 2004; Amorim et al. 2005) e do Sudeste do país (Leitão-Filho 1982; Peixoto \& Gentry 1990; Guedes-Bruni 1998). Tal padrão corrobora as observações de Prance et al. (1979) e Gentry (1995) sobre a importância dessas famílias nas florestas neotropicais.

Com relação à distribuição por estrato $\mathrm{e}$ habitat, as espécies emergentes mais comuns foram Albizia polycephala, Copaifera trapezifolia, Eriotheca crenulaticalyx e Manilkara rufula. No 


\section{Similaridade Florística}

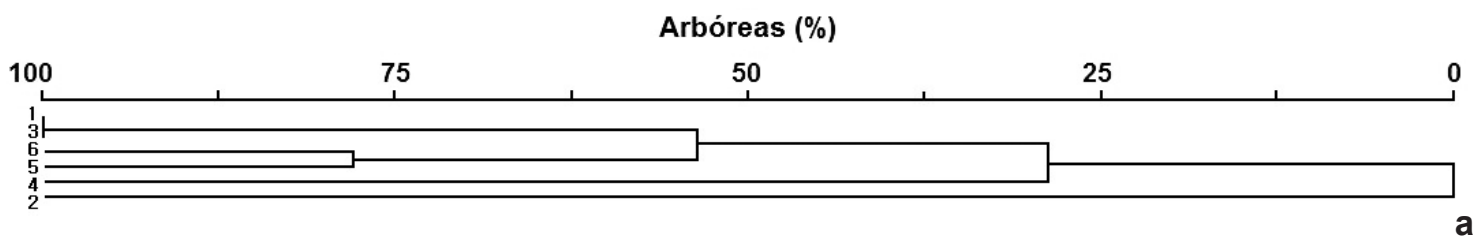

Arbusto/subarbusto (\%)

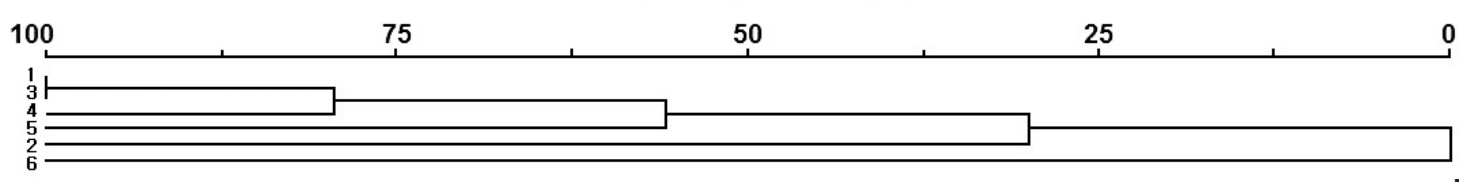

Herbácea/trepadeira (\%)

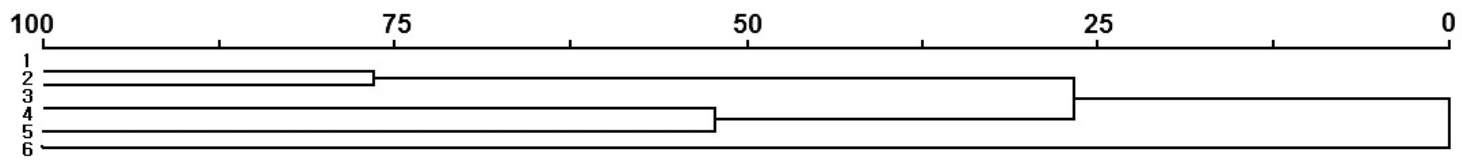

Figura 2 - Análise de similaridade florística do componente arbóreo (a), arbustivo/subarbustivo (b) e herbáceo/ trepadeira (c) entre a área de estudo e outras de Pernambuco (1 - Brejo da Madre de Deus; 2 - Floresta; 3 - Caruaru; 4 - Bonito; 5 - São Vicente Férrer; 6 - São Lourenço da Mata).

Figure 2-Analysis of floristic similarity of the tree component (a), shrub/shrublet (b) and herb/climbing (c) between the study area and other of Pernambuco (1 - Brejo da Madre de Deus; 2 -Floresta; 3 - Caruaru; 4 - Bonito; 5 - São Vicente Férrer; 6 - São Lourenço da Mata).

dossel, se destacaram Bowdichia virgilioides, Inga subnuda, Buchenavia capitata, Guapira nitida, Schefflera morototoni, Simarouba amara, entre outras. No sub-dossel destaque para Banara brasiliensis, Casearia sylvestris, Machaerium hirtum, Nectandra cuspidata, Eugenia ligustrina, E. punicifolia e Myrcia fallax. Nas bordas das matas foram comuns Byrsonima crispa, Erythroxylum citrifolium, Inga marginata, Miconia caudigera e M. rimalis e Myrcia sylvatica. Em afloramentos rochosos, situados nas áreas mais elevadas, Miconia rubiginosa, Eugenia obtusifolia e Marlierea clausseniana foram as mais comuns. Com exceção de Albizia polycephala, Buchenavia capitata, E. punicifolia e Myrcia fallax, que tem ocorrência também na área Floresta, um Brejo de Altitude do sertão (Rodal e Nascimento 2002), as demais espécies têm distribuição no domínio atlântico, do agreste ao litoral do Estado (Rodal et al. 2005a, b; Ferraz \& Rodal 2008; Rodal \& Sales 2008).
A análise de similaridade florística do hábito arbóreo (Fig. 2a) confirma estas observações, indicando que lista de Brejo da Madre de Deus tem alto nível de semelhança com as florestas mais próximas da costa atlântica como Caruaru (Rodal \& Sales 2008), São Vicente Férrer (Ferraz \& Rodal 2008) e de São Lourenço da Mata (Rodal et al. 2005a), indicando uma ligação florística com a flora atlântica. Estes resultados corroboram as afirmações de Rodal et al. (2005a) sobre o efeito da continentalidade e sua influência na formação de conjuntos florísticos dos brejos de altitude de Pernambuco, reforçando estudos em escala continental e regional que mostraram que a composição de espécies de árvores varia em resposta ao clima e a biogeografia, determinando a distribuição das espécies arbóreas na paisagem tropical de acordo com suas tolerâncias e adaptações específicas (Hall \& Swaine 1976, 1981; Lieberman et al. 1985; Hubbell \& Foster 1986; Chazdon \& Denslow 2002). 
Floristica de uma floresta montana de Pernambuco, Brasil

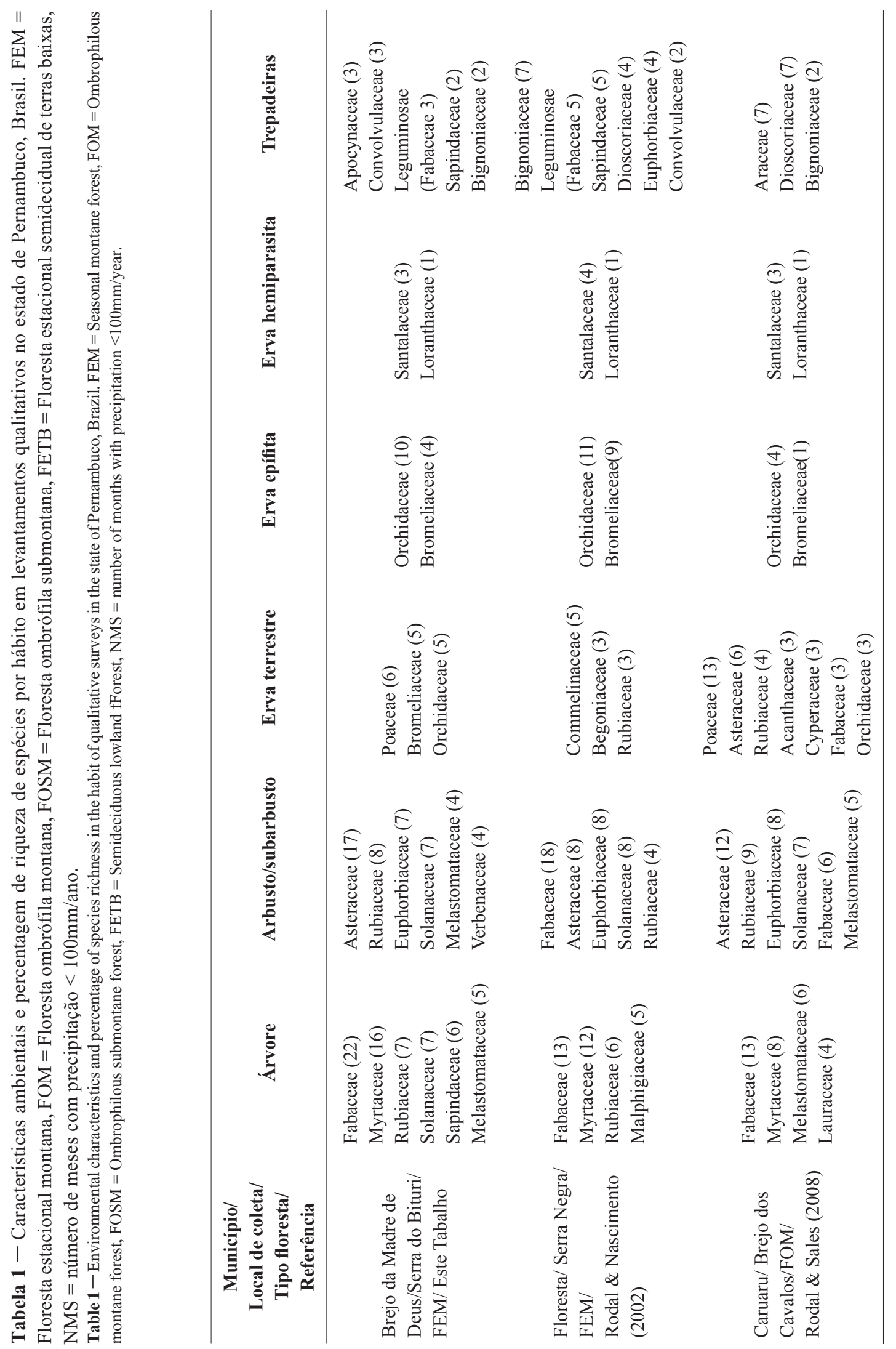




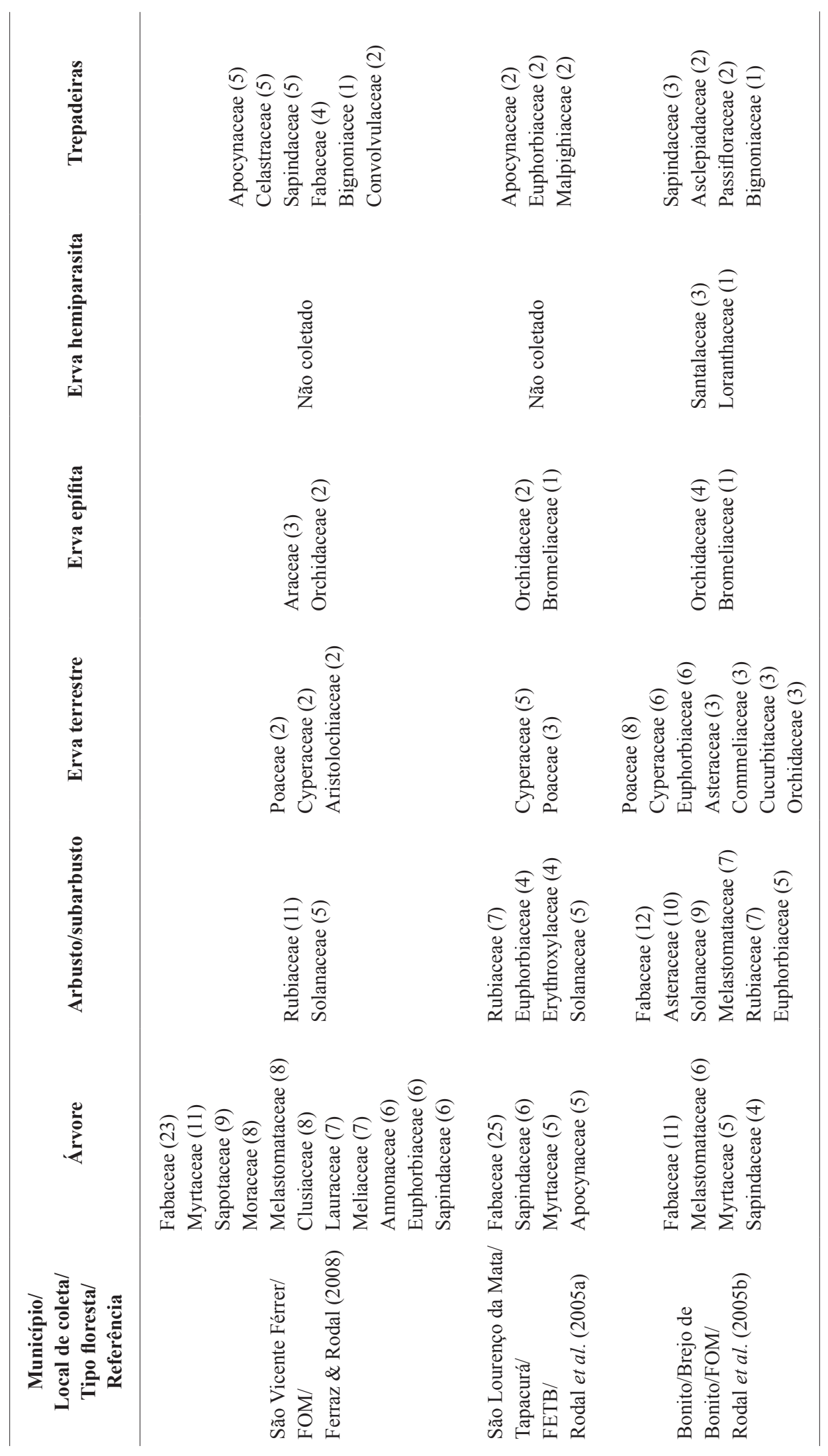


Arbustos/subarbustos - Cerca de 24\% das espécies apresentaram esse hábito, com destaque para Asteraceae com 17 espécies, seguida por Rubiaceae (8 spp.), Euphorbiaceae e Solanaceae (7 spp. cada) e Melastomataceae (4 spp.). Com exceção de Melastomataceae, estas famílias também foram citadas como importantes em riqueza (Tab. 1) nas florestas de Caruaru (Rodal \& Sales 2008) e Bonito (Rodal et al. 2005b), porém com composição florística distinta.

Como observado na área de estudo Tabarelli \& Mantovani (1999), Ivanauskas et al. (2001) e Zipparro et al. (2005) também citaram Rubiaceae, Melastomataceae e Solanaceae como as famílias mais ricas em espécies do componente arbustivo em áreas de Floresta Atlântica. Enquanto Asteraceae e Euphorbiaceae (gêneros Cnidoscolus e Croton) foram citadas com maior riqueza em áreas de Caatinga ou no contato Caatinga - Brejo de Altitude (Melo \& Sales 2008; Rodal \& Sales 2008 e Lucena 2000, respectivamente).

A análise de similaridade desses hábitos (Fig. 2b) indicou maior semelhança florística da área de estudo com as florestas montanas de Caruaru e Bonito (mais próximos da costa atlântica), compartilhando cerca 50\% das espécies de Rubiaceae (por exemplo, Psychotria carthagenensis, P. schlechtendaliana), Solanaceae (Solanum baturitense e $S$. paniculatum) e Melastomataceae (Clidemia debilis e C. hirta) e cerca de 30\% de Asteraceae (Baccharis oxyodonta e Verbesina macrophylla). Assim como ocorreu no hábito arbóreo, a semelhança da área de estudo em termos de arbustos/subarbustos com as florestas montanas mais costeiras reforça a idéia de ligação de algumas florestas montanas com a flora atlântica (Rodal et al. 2005), que poderia ser justificada principalmente pela importância em riqueza das famílias Rubiaceae e Solanaceae na floresta atlântica (Tabarelli \& Mantovani 1999) e, de forma geral, nas florestas neotropicais montanas (Gentry 1982, 1988, 1990).

Foi possível identificar a preferência de algumas espécies por habitats específicos. Por exemplo, no interior da mata foram destaque: Aureliana fasciculata, Brunfelsia uniflora, Chiococca alba e Palicourea crocea; nas áreas de borda: Acacia paniculata, Alternanthera brasiliana, Psychotria sessilis, Solanum paraibanum, S. rhytidondrum, Verbesina macrophylla e Vernonia andrade-limae, e nos afloramentos rochosos: Paliavana tenuifolia, Psychotria schlechtendaliana e Tibouchina heteromalla.
Ervas terrestres - Esse grupo apresenta 25 espécies (cerca de 10\% da flora total), destacandose Poaceae ( 8 spp.), Bromeliaceae (5 spp.) e Asteraceae (4 spp.). Nos levantamentos listados na Tabela 2, Poaceae figura como o táxon com maior riqueza de espécies, exceto em Floresta e São Lourenço da Mata. Das espécies amostradas, nove foram compartilhadas com pelo menos uma das áreas listadas na Tabela 2, sendo comuns nas bordas da mata e margens da estrada.

As espécies não compartilhadas, como Dyckia limae e Vriesea limae, endêmicas dos afloramentos rochosos de Pernambuco (SiqueiraFilho 2002), formavam moitas juntamente com Epidendrum cinnabarinum, Eragrostis neesii, Melinis repens, Melocactus zehntneri, Oncidium barbatum e Vrisea friburgensis. A formação de moitas de Bromeliaceae, Poaceae e Orchidaceae, associado espécies de outras famílias foi um padrão também citado por Barthlott et al. (1996) e Porembski et al. (1997) em afloramentos rochosos sulamericanos.

No interior dos fragmentos não era comum a presença de ervas, com exceção das áreas de clareiras. Nas bordas florestais foram encontradas espécies como Conocliniopsis prasiifolia, Erechtites valerianifolia e Vernonia acutangula, nas áreas de nascentes ou próximos a cursos d'água predominavam agrupamentos de Heliconia psittacorum. A esse respeito, Grime (1979) observou que a presença e a riqueza de espécies nos diferentes habitats do "chão" das florestas dependeria da intensidade luminosa que ali chega.

Ervas epífitas - Neste grupo foram registradas 16 espécies, correspondendo a 6,3\% da flora total, com destaque para Orchidaceae (10 espécies) e Bromeliaceae (4), confirmando as observações de Gentry \& Dodson (1987), que descreveram a elevada riqueza de Orchidaceae epífitas, superando todos os grupos de plantas em número de espécies nos trópicos e subtrópicos.

Em média, os levantamentos citados na Tabela 2 tiveram 4\% de epífitas, havendo claramente uma maior riqueza de espécies na área de estudo e em Floresta, mais secas se comparadas àqueles brejos mais próximos do litoral. Tais resultados contrariam as observações de Gentry (1995), que ressalta que esse grupo tem maior riqueza nas florestas úmidas que nas secas. É possível levantar duas hipóteses para essa maior riqueza nesses Brejos de Altitude: 1) tanto 


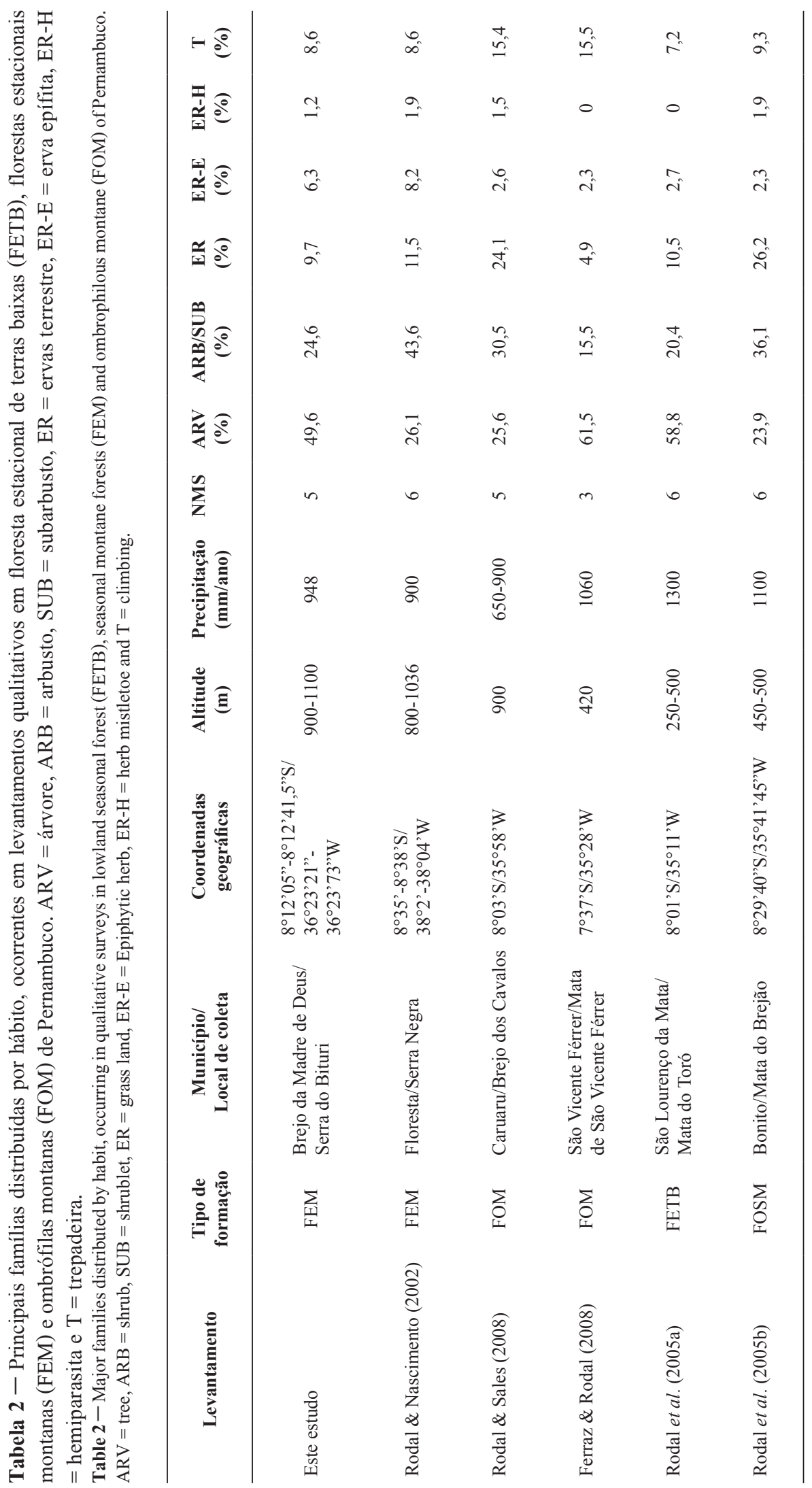


na área de estudo, quanto em Floresta (Rodal \& Nascimento 2002), houve um maior esforço de coleta ou 2) que haja maior riqueza de epífitas nessas florestas estacionais de maior altitude, em função da maior nebulosidade ou "precipitação oculta". A esse respeito, especialmente orquídeas e bromélias, apresentam adaptações morfológicas para absorção de água e nutrientes nos estratos mais altos da floresta, o que pode representar uma vantagem em ambientes caracterizados pela alta nebulosidade que, segundo Vogelmann (1973), é o fator responsável pela existência e manutenção das florestas montanas.

No interior do fragmento foi comum a presença de Cattleya labiata e Prosthechea fragans, Tillandsia cf. juncea, $T$. tenuifolia e $T$. usneoides. Algumas espécies como Rhipsalis cereuscula utilizavam como suporte árvores de grande porte como Eriotheca crenulaticalyx enquanto Peperomia tetraphylla era usualmente observada em troncos em decomposição.

Ervas hemiparasitas - Hábito com quatro espécies (1,5\%), sendo Loranthaceae e Santalaceae os únicos representantes. São plantas muito comuns em borda de mata, como também registrado em Floresta, Caruaru e Bonito, com as mesmas famílias e, quase sempre, o mesmo número de espécies (Tab. 1). Struthanthus marginatus ocorreu nos levantamentos realizados em Caruaru e Bonito, enquanto Phoradendron piauhyanum e P. crassifolium, ocorreram em Floresta (Rodal \& Nascimento 2002) e Bonito, respectivamente.

Trepadeiras - Foram registradas 22 espécies, entre trepadeiras lenhosas e herbáceas, correspondendo a 8,6\% das espécies da área de estudo. Em média, os levantamentos citados na Tabela 2 têm 10\% de trepadeiras, valor inferior aos $20 \%$ relatado por Gentry (1991), para florestas tropicais. Com base na literatura consultada, apenas as florestas mais úmidas estudadas por Rodal \& Sales (2008) e Ferraz \& Rodal (2008) têm acima de $15 \%$ de trepadeiras. Fatores como clima, altitude, solo e posição geográfica têm sido relatados como importantes para explicar diferenças de composição e abundância deste hábito (Gentry 1988).

De um modo geral, o total de espécies trepadeiras em florestas pernambucanas é baixo, quando comparado com os levantamentos no Sudeste do Brasil realizados por Hora \& Soares (2002), Udulutsch et al. (2004), Rezende \& Ranga (2005) e Tibiriçá et al. (2006). Todavia, vale ressaltar que a diferença no esforço amostral não permite maiores considerações, uma vez que todos estes estudos enfocaram exclusivamente as trepadeiras (lenhosas e herbáceas). Considerando que pouco se conhece a respeito das variações florísticas deste componente em Pernambuco, é importante que estudos que priorizem este componente sejam realizados com maior intensidade, principalmente por este apontar o estado de conservação das florestas (Schnitzer \& Bongers 2002).

As famílias com maior riqueza de espécies foram Apocynaceae, Bignoniaceae, Convolvulaceae e Fabaceae com três espécies cada, seguida por Sapindaceae e Malpighiaceae (duas cada). Estas famílias também são importantes em Floresta e São Vicente Férrer, assim como em florestas estacionais no Sudeste do Brasil (Udulutsch et al. 2004). Tanto na área de estudo como em outras florestas, percebe-se que a maioria das espécies de trepadeiras concentra-se num pequeno número de famílias, como observou Gentry (1991), destacando Bignoniaceae como a família mais rica nesse hábito em florestas neotropicais.

Quanto às espécies compartilhadas, a maior semelhança ocorreu com Caruaru e Bonito, com quatro espécies cada, dentre elas: Mandevilla dardanoi, Merremia macrocalyx e Paullinia trigonia, e a menor semelhança com São Lourenço da Mata, com apenas duas espécies (Mandevilla scabra e Tetrapteris mucronata). A primeira espécie é considerada endêmica dos inselbergues ocorrentes na formação geológica Planalto da Borborema, no nordeste do Brasil (Sales et al. 2006). Mandevilla scabra, ao contrário, tem ampla distribuição geográfica no estado de Pernambuco, ocorrendo em capoeiras e bordas de Floresta Atlântica, sendo restrita a áreas de maior altitude no agreste do estado.

Em relação à distribuição nos diferentes ambientes, Smilax syphilitica destacou-se no interior da mata e Dioclea guianensis, Jacquemontia martii, Pyrostegia venusta e Serjania ichthyoctona foram comuns na borda da mata.

A análise de similaridade do conjunto formado por ervas terrestres, epifíticas, hemiparasitas e trepadeiras (Fig. 2c) indicou que a área de estudo tem maior similaridade florística com Floresta (Rodal \& Nascimento 2002). Esta 
semelhança pode ser justificada pelo levantamento igualmente detalhado desses hábitos nas duas áreas, ou ainda, pelo estado de conservação destas florestas (Rodal \& Sales 2008). O reduzido número de levantamentos avaliando esses componentes dificulta maiores discussões, necessárias para viabilizar estratégias de conservação para estas florestas ameaçadas.

\section{Agradecimentos}

Ao CNPq a concessão da bolsa de mestrado à primeira autora, de produtividade da segunda autora e a bolsa de IC ao terceiro autor. À Fundação O Boticário de Proteção à Natureza (proc. 0345981) e ao WWF-Brasil (proc. 193-200), $\mathrm{o}$ apoio financeiro à pesquisa. Aos Srs. Reginaldo Araújo e Jurandir Amorim, proprietários da fazenda Bituri, o apoio à realização deste estudo.

\section{Referências}

Amorim, A.M.; Jardim, J.G.; Clifton, B.C.; Fiaschi, P.; Thomas, W.W. \& Carvalho, A.M.V. 2005. The vascular plants of a forest fragment in southern Bahia, Brazil. Sida 21: 1727-1752.

Andrade, G.O. \& Lins, R.C. 1965. Introdução a morfoclimatologia do nordeste do Brasil. Arquivo do Instituto de Ciência da Terra 4: 17-28.

Andrade-Lima, D. 1966. Esboço fitoecológico de alguns "brejos" de Pernambuco. Boletim Técnico do Instituto de Pesquisa Agronômicas de Pernambuco 8: 3-10.

Andrade-Lima, D. 1981. The caatinga dominium. Revista Brasileira de Botânica 4: 149-163.

APG III. 2009. An update of the Angiosperm Phylogeny Group classification for the orders and families of flowering plants: APG III. Botanical Journal of the Linnean Society 161: 105-121.

Barthlott, W.; Porembski, S.; Szarzynski, J. \& Mund, J.P. 1996. Phytogeography and vegetation of Tropical Inselbergs. In: Guillaumet, J.L.; Belin, M. \& Puig, H. (eds.). Phytogéographie tropicale: réalités et perspectives. Paris, Orstom (Colloques et Séminaires). Phytogéographie Tropicale: Colloque International en hommage au Professeur Raymond Schnell. Pp. 15-24.

Cestaro, L.A. \& Soares, J.J. 2004. Variações florística e estrutural e relações fitogeográficas de um fragmento de floresta decídua no Rio Grande do Norte. Acta Botanica Brasilica 18: 203-218.

Chazdon, R.L. \& Denslow, J.S. 2002. Floristic composition and species richness. In: Chazdon, R.L. \& Whitmore, T.C. (eds). Foundations in tropical forest biology: classic papers with commentaries. University of Chicago Press. Pp. 513-522.
Ferraz, E.M.N. \& Rodal, M.J.N. 2006. Caracterização fisionômica-estrutural de um remanescente de floresta ombrófila montana de Pernambuco, Brasil. Acta Botanica Brasilica 20: 911-926.

Ferraz, E.M.N. \& Rodal, M.J.N. 2008. Floristic characterization of a remnant ombrophilous montane forest at São Vicente Férrer, Pernambuco, Brazil. Memoirs of the New York Botanical Garden 100: 468-510.

Filgueiras, T.S.; Brochado, A.L.; Nogueira, P.E. \& Guala Ii, G.F. 1994. Caminhamento: um método expedito para levantamentos florísticos qualitativos. Cadernos de Geociências 12: 39-43

Font-Quer, P. 2000. Diccionario de Botánica. Ediciones Península, Barcelona. 1244p.

Gentry, A.H. 1982. Neotropical floristic diversity: phytogeographical connections between Central and South America, Pleistocene climatic fluctuations, or an accident of the andean orogeny? Annals of the Missouri Botanical Garden 69: 557-593.

Gentry, A.H. 1988. Changes in plant community diversity and floristic composition on environment and geographical gradients. Annals of the Missouri Garden 75: 1-34.

Gentry, A.H. 1990. Floristic similarities and differences between Southern Central America and upper and Central Amazonia. In: Gentry, A.H. (ed.). Four neotropical rain forests. Yale University Press, London. Pp. 141-160.

Gentry, A.H. 1991. The distribution and evolution of climbing plants. In: Putz, F.E. \& Mooney, H.A. (eds.). The biology of vines. Cambridge University Press, Cambridge. Pp. 3-53.

Gentry, A.H. 1995. Diversity and floristic composition of neotropical dry forests. In: Bullock, S.H.; Mooney, H.A. \& Medina, E. (eds.). Seasonally dry forests. Cambridge University Press, Cambridge. Pp. 146-194.

Gentry, A.H. \& Dodson, C.H. 1987. Diversity and biogeography of neotropical vascular epiphytes. Annals of the Missouri Botanical Garden 74: 205-233.

Grime, J.P. 1979. Plant strategies and vegetation processes. John Wiley \& Sons, Chichester. 247p.

Guedes, M.L.S. 1998. A vegetação fanerogâmica da Reserva Ecológica de Dois Irmãos. In: Machado, I.C.S.; Lopes, A.V. \& Porto, K.C. (orgs.). Reserva Ecológica de Dois Irmãos. Estudos em um remanescente de mata atlântica em área urbana (Recife-Pernambuco-Brasil). Editora Universitária, Universidade Federal de Pernambuco, Recife. Pp. 157-172.

Guedes-Bruni, R.R. 1998. Composição, estrutura e similaridade florística de dossel em seis unidades fisionômicas de Mata Atlântica no Rio de Janeiro. Tese de Doutorado. Universidade de São Paulo, São Paulo. 231p. 
Hall, J.B. \& Swaine, M.D. 1976. Classification and ecology of closed canopy forest in Ghana. Journal of Ecology 64: 913-951.

Hall, J.B. \& Swaine, M.D. 1981. Distribution and ecology of vascular plants in a tropical rainforest: Forest vegetation in Ghana. W. Junk, deen Haag. $383 p$.

Hora, R.C. \& Soares, J.J. 2002. Estrutura fitossociológica da comunidade de lianas em uma floresta estacional semidecidual na Fazenda Canchim, São Carlos, SP. Revista Brasileira de Botânica 25: 323-329.

Hubbell, S.P. \& Foster, R.B. 1986. Canopy gaps and the dynamics of a neotropical forest. In: Crawley, M.J. (ed.). Plant ecology. Blackwell Scientific, Oxford. Pp. 77-96.

IBGE - Instituto Brasileiro de Geografia e Estatística. 1993. Mapa de vegetação do Brasil. Escala 1:5.000.000. 2a ed. IBGE, Rio de Janeiro. 61p.

IPNI - The International Plant Names Index. Disponível em $<$ http://www.ipni.org $>$. Acesso em 27 Ago 2010 .

Ivanauskas, N.M.; Monteiro, R. \& Rodrigues, R.R. 2001. Levantamento florístico de trecho de floresta Atlântica em Pariquera-Açu, São Paulo, Brasil. Naturalia 26: 97-129.

Leitão-Filho, H.F. 1982. Aspectos taxonômicos das florestas do estado de São Paulo. Sivicultura em São Paulo 16: 197-206.

Lieberman, M.; Lieberman, D.; Hartshorn, G.S. \& Peralta, R. 1985. Small-scale altitudinal variation in lowland wet tropical forest vegetation. Journal of Ecology 73: 505-516.

Lourenço, C.E.L. \& Barbosa, M.R.V. 2003. Flora da fazenda Ipuarana, Lagoa Seca, Paraíba (Guia de campo). Revista Nordestina de Biologia 17: 23-58.

Lucena, M.F.A. 2000. Estudos taxonômicos do gênero Croton L. (Crotonoideae -Euphorbiaceae) nas zonas do litoral e da mata do estado de Pernambuco - Brasil. Dissertação de Mestrado. Universidade Federal Rural de Pernambuco, Recife. 131p.

McCune, B. \& Mefford, M.J. 1999. PC-ORD version 4.0, multivariate analysis of ecological data. Users guide. MjM Software Design, Glaneden Beach. $148 \mathrm{p}$.

Melo, A.L \& Sales, M.F. 2008. Estudos taxonômicos sobre o gênero Cnidoscolus Pohl (CrotonoideaeEuphorbiaceae) no estado de Pernambuco. Acta Botanica Brasilica 22: 806-827.

Mori, S.A.; Silva, L.A.M.; Lisboa, G. \& Coradin, L. 1989. Manual de manejo do herbário fanerogâmico. Centro de Pesquisas do Cacau, Ilhéus. 104p.

Moura, F.B.P. \& Sampaio, E.V.S.B. 2001. Flora lenhosa de uma mata serrana semidecídua em Jataúba, Pernambuco. Revista Nordestina de Biologia 15: 77-89.

Nascimento, L.M. \& Rodal, M.J.N. 2008. Fisionomia e estrutura de uma floresta estacional montana do maciço da Borborema, Pernambuco - Brasil. Revista Brasileira de Botânica 31: 27-39.

Oliveira-Filho, A.T.; Jarenkon, J.A. \& Rodal, M.J.N. 2006. Floristic relationships of seasonally dry forests of Eastern South America based on tree species distribution patterns. In: Pennington, R.T.; Lewis, G.P. \& Ratter, J.A. (eds.). Neotropical savannas and seasonally dry forests: plant diversity, biogeography, and conservation. Taylor \& Francis Group, Boca Raton. Pp. 159-192.

Peixoto, A.L. \& Gentry, A.H. 1990. Diversidade e composição florística da mata de tabuleiro na Reserva Florestal de Linhares (Espírito Santo, Brasil). Revista Brasileira de Botânica 13: 19-25.

Prance, G.T. 1979. The taxonomic and phytogeography of Chrysobalanaceae of the atlantic coastal forest of Brazil. Revista Brasileira de Botânica 2: 19-39.

Porembski, S.; Seine, R. \& Barthlott, W. 1997. Inselberg vegetation and the biodiversity of granite outcrops. Journal of the Royal Society of Western Australia 80: 193-199.

Rezende, A.A. \& Ranga, N.T. 2005. Lianas da Estação Ecológica do Noroeste Paulista, São José do Rio Preto/Marissol, SP, Brasil. Acta Botanica Brasilica 19: 273-279.

Richards, P.W. 1996. The tropical rain forest: an ecological study. Cambridge University Press, Cambridge. 575p.

Rodal, M.J.N.; Barbosa, M.R.V. \& Thomas, W.W. 2008. Do the seasonal forests in northeastern Brazil represent a single floristic unit? Brazilian Journal of Biology 68: 631-637.

Rodal, M.J.N.; Lucena, M.F.A.; Andrade, K.V.S.A. \& Melo, A.L. 2005a. Mata do Toró: uma floresta estacional semidecidual de terras baixas no nordeste do Brasil. Hoehnea 32: 283-294.

Rodal, M.J.N. \& Nascimento, L.M. 2002. Levantamento florístico da floresta serrana da reserva biológica de Serra Negra, microrregião de Itaparica, Pernambuco, Brasil. Acta Botanica Brasilica 16: 481-500.

Rodal, M.J.N. \& Nascimento, L.M. 2006. The arboreal component of a dry forest in northeastern Brazil. Brazilian Journal of Biology 66: 479-492.

Rodal, M.J.N. \& Sales, M.F. 2008. Panorama of the montane forests. Memoirs of the New York Botanical Garden 100: 535-553.

Rodal, M.J.N.; Sales, M.F.; Silva, M.J.S. \& Silva, A.G. 2005b. Flora de um brejo de altitude na escarpa oriental do planalto da Borborema, PE, Brasil. Acta Botanica Brasilica 19: 843-858.

Sales, M.F.; Kinoshita, L. S. \& Simões, A. 2006. Eigth new species of Mandevilla Lindley (Apocynaceae: Apocynoideae) from Brazil. Novon 16: 112-128.

Sales, M.F.; Mayo, S.J. \& Rodal, M.J.N. 1998. Plantas vasculares das florestas serranas de Pernambuco. Um checklist da flora ameaçada dos brejos de altitude. Imprensa Universitária, 
Universidade Federal Rural de Pernambuco, Recife. 130p.

Schnitzer, S.A. \& Bongers, F.A. 2002. The ecology of lianas and their role in forests. Trends in Ecology \& Evolution 17: 223-230.

Silva, D.G.; Melo, R.F.T.; Corrêa, A.C.B. 2009. A influência da densidade de drenagem na interpretação da evolução geomorfológica do complexo de tanques do município de Brejo da Madre de Deus - Pernambuco, nordeste do Brasil. Revista de Geografia 26: 294-306.

Siqueira, S.R.; Rodal, M.J.N.; Lins-e-Silva, A.C.B. \& Melo, A.L. 2001. Physiognomy, structure, and floristics in an area of Atlantic Forest in Northeast Brazil. Dissertationes Botanicae 346: 11-17.

Siqueira-Filho, J.A. 2002. Bromélias em Pernambuco: diversidade e aspectos conservacionistas. In: Tabarelli, M. \& Silva, J.M.C. (orgs.). Diagnóstico da Biodiversidade de Pernambuco. Secretaria de Ciência, Tecnologia e Meio Ambiente, Editora Massangana, Recife. Pp. 218-225.

Tabarelli, M. \& Mantovani, W. 1999. A riqueza de espécies arbóreas na floresta atlântica de encosta no estado de São Paulo (Brasil). Revista Brasileira de Botânica 22: 217-223.

Tabarelli, M. \& Santos, A.M.M. 2004. Uma breve descrição sobre a história natural dos brejos nordestinos. In: Pôrto, K.C.; Cabral, J.J.P. \& Tabarelli, M. (orgs.). Brejos de Altitude em Pernambuco e Paraíba: História natural, ecologia e conservação. Ministério do Meio Ambiente, Brasília. Pp. 99-110.

Tibiriçá, Y.J.A.; Coelho, L.F.M. \& Moura, L.C. 2006. Florística de lianas em um fragmento de floresta estacional semidecidual, Parque Estadual de Vassununga, Santa Rita do Passa Quatro, SP, Brasil. Acta Botanica Brasilica 20: 339-346.

Udulutsch, R.G.; Assis, M.A. \& Picchi, D. 2004. Florística de trepadeiras numa floresta estacional semidecídua, Rio Claro - Araras, Estado de São Paulo, Brasil. Revista Brasileira de Botânica 27: 125-134.

Vasconcelos Sobrinho, J. 1971. As regiões naturais do Nordeste, o meio e a civilização. CONDEPE, Recife. 441p.

Veloso, H.P.; Rangel-Filho, A.L.R. \& Lima, J.C.A. 1991. Classificação da vegetação brasileira, adaptada a um sistema universal. IBGE, Rio de Janeiro. 123p.

Vogelmann, H.W. 1973. Fog precipitation in the cloud forests of Eastern Mexico. BioScience 23: 96-100.

Zipparro, V.B.; Guilherme, F.A.G.; Almeida-Scabbia, R. \& Morellato, P.C. 2005. Levantamento florístico de Floresta Atlântica no sul do Estado de São Paulo, Parque Estadual Intervales, Base Saibadela. Biota Neotropica 5: 1-24. 


\section{Florística de uma floresta estacional no Planalto da Borborema, nordeste do Brasil}

Floristic of seasonal forest in the Borborema Plateau, northeastern Brazil

Ladivania Medeiros do Nascimento, Maria Jesus Nogueira Rodal \& Alexandre Gomes da Silva

Apêndice 1 - Famílias e espécies registradas na Serra do Bituri, Brejo da Madre de Deus, Pernambuco, com nome, número de coletor $\left(\mathrm{N}^{\circ}\right)$, hábito $(\mathrm{ARV}=$ árvore, $\mathrm{ARB}=$ arbusto, $\mathrm{SUB}=$ subarbusto, $\mathrm{ER}=$ erva terrestre, $\mathrm{ER}-\mathrm{E}=\mathrm{erva}$ epífita, ER-H = erva hemiparasita e $\mathrm{T}=$ trepadeira). Especialistas que auxiliaram na determinação dos taxa estão listados após cada família.

Appendix 1 - Families and species found in the Serra do Bituri, Brejo da Madre de Deus, Pernambuco, with name, collector number (No.), habit $(\mathrm{ARV}=$ tree, $\mathrm{ARB}=$ shrub, $\mathrm{SUB}=$ shrublet, $\mathrm{ER}=$ grass land, ER-E = Epiphytic herb, $\mathrm{ER}-\mathrm{H}=$ herb mistletoe and $\mathrm{T}=$ climbing). Experts who have helped in determining the rate are listed after each family.

\begin{tabular}{llr}
\hline FAMÍLIA/ESPÉCIE & COLETOR/No & HÁBITO \\
\hline $\begin{array}{l}\text { Acanthaceae } \\
\text { Ruellia devosiana Makoy ex E. Murr. }\end{array}$ & L.M.Nascimento 430 & SUB \\
$\begin{array}{l}\text { Amaryllidaceae } \\
\text { Hippeastrum } \text { sp. }\end{array}$ & A.G. da Silva 187 & ER
\end{tabular}

\section{Annonaceae}

Guatteria australis A. St.-Hil.

L.M. Nascimento 131

ARV

Apocynaceae (M.F. Sales - PEUFR; A. Rapini - UEFS)

Allamanda blanchetii A. DC.

A.G. da Silva 54

$\mathrm{ARB}$

Aspidosperma parvifolium A. DC.

L.M. Nascimento 423

ARV

Aspidosperma sp.

L.M. Nascimento 309

ARV

Mandevilla dardanoi M.F.Sales

L.M. Nascimento 509

M. scabra (Hoffmanns. ex Roem. \& Schult.) K. Schum.

L.M. Nascimento 344

$\mathrm{T}$

M. tenuifolia (J.C. Mikan) Woodson

L.M. Nascimento 215

Orthosia congesta (Vell.) Decne.

A.G. da Silva 223

Aquifoliaceae (M. Groppo Jr. - SPFR)

Ilex conocarpa Reissek

L.M. Nascimento 200

I. sapotifolia Reissek

L.M. Nascimento 199

* Ilex sp. (espécie nova)

Araliaceae

Schefflera morototoni (Aubl.) Maguire, Steyerm. \& Frodin

Arecaceae

Bactris pickelii Burret

Asteraceae (R. Pereira - IPA; N. Roque - HUEFS)

Achyrocline satureioides (Lam.) DC. 


\begin{tabular}{|c|c|c|}
\hline FAMÍLIA/ESPÉCIE & COLETOR/No & HÁBITO \\
\hline Alternanthera brasiliana (L.) Kuntze & A.M. da Silva 7 & ARB \\
\hline Baccharis aff. calvescens DC. & L.M. Nascimento 153 & $\mathrm{ARB}$ \\
\hline B. oxyodonta DC. & A.G. da Silva 6 & SUB \\
\hline B. rhexioides Kunth. & L.M. Nascimento 263 & SUB \\
\hline Baccharis sp. & A.M. da Silva 14 & ER \\
\hline Conocliniopsis prasiifolia (DC.) R.M. King \& H. Rob. & A.G. da Silva 6 & ER \\
\hline Erechtites valerianaefolia (Wolf.) DC. & L.M. Nascimento 440 & ER \\
\hline Gochnatia oligocephala (Gardner) Cabrera & L.M. Nascimento 350 & ARB \\
\hline Mikania cordifolia (L.f.) Willd. & L.M. Nascimento 268 & $\mathrm{~T}$ \\
\hline Solidago microglossa DC. & L.M. Nascimento 356 & SUB \\
\hline Struchium sparganophorum (L.) Kuntze & L.M. Nascimento 197 & ARV \\
\hline Tilesia baccata (L.) Pruski & A.M. da Silva 15 & ARB \\
\hline Tixis antimenorrhoea (Schrank) Kuntze & A.M. da Silva 5 & $\mathrm{ARB}$ \\
\hline Vanillosmopsis arborea (Gardner) Baker & A.G. da Silva 132 & ARB \\
\hline Verbesina macrophylla (Cass.) S.F. Blake & A.M. da Silva 58 & ARB \\
\hline Vernonia acutangula Gardner & L.M. Nascimento 441 & ER \\
\hline V. andrade-limae G.M. Barroso & A.M. da Silva 4 & $\mathrm{ARB}$ \\
\hline$V$. cf. eremophila Mart. ex DC. & A.G. da Silva 330 & SUB \\
\hline V. ferruginea Less. & A.M. da Silva 35 & ARB \\
\hline$V$. aff. riedelii Sch. Bip ex Baker & A.G. da Silva 137 & ARB \\
\hline Wedelia sp. & A.G. da Silva 255 & ARB \\
\hline \multicolumn{3}{|l|}{ Bignoniaceae } \\
\hline Lundia cordata (Vell.) A. DC. & A.G. da Silva 68 & $\mathrm{~T}$ \\
\hline Pyrostegia venusta (Ker Gawl.) Miers & C.A.M. de Oliveira 2 & $\mathrm{~T}$ \\
\hline Tabebuia impetiginosa (Mart. ex DC.) Standl. & C.A.M. de Oliveira 48 & ARV \\
\hline T. cf. serratifolia (Vahl) G. Nicholson & C.A.M. de Oliveira 47 & ARV \\
\hline Bignoniaceae A & L.M. Nascimento 401 & $\mathrm{~T}$ \\
\hline \multicolumn{3}{|l|}{ Boraginaceae (J.I. Melo - PEUFR) } \\
\hline Cordia curassavica (Jacq.) Roem. \& Schult. & L.M. Nascimento 324 & ARB \\
\hline C. sellowiana Cham. & L.M. Nascimento 155 & ARV \\
\hline C. aff. superba Cham. & L.M. Nascimento 115 & ARV \\
\hline C. trichotoma (Vell.) Arráb. ex Steud. & L.M. Nascimento 422 & ARV \\
\hline Tournefortia paniculata Vent. & L.M. Nascimento 118 & $\mathrm{ARB}$ \\
\hline \multicolumn{3}{|l|}{ Brassicaceae } \\
\hline Capparis flexuosa (L.) L. & L.M. Nascimento 193 & ARB \\
\hline \multicolumn{3}{|c|}{ Bromeliaceae (M.G. Wanderley - HSP; G.M. Souza - TEPB) } \\
\hline Dyckia limae L.B. Sm. & L.M. Nascimento $511 \mathrm{~b}$ & ER \\
\hline Orthophytum disjunctum L.B. Sm. & A.G. da Silva 192 & ER \\
\hline Portea leptantha Harms & L.M. Nascimento 315 & ER \\
\hline
\end{tabular}




\begin{tabular}{|c|c|c|}
\hline FAMÍLIA/ESPÉCIE & COLETOR/No & HÁBITO \\
\hline Tillandsia gardneri Lindl. & L.M. Nascimento 517 & ER-E \\
\hline T. juncea (Ruiz \& Pav.) Poir. & A.G. da Silva 238 & ER-E \\
\hline T. tenuifolia $\mathrm{L}$. & L.M. Nascimento 445 & ER-E \\
\hline T. usneoides (L.) L. & L.M. Nascimento 288 & ER-E \\
\hline Vriesea friburgensis Mez. & A.G. da Silva 406 & ER \\
\hline V. limae L.B. Sm. & A.G. da Silva 401 & ER \\
\hline \multicolumn{3}{|l|}{ Cactaceae (D. Zappi - RBG) } \\
\hline Melocactus zehntneri (Britton \& Rose) Luetzelb. & A.G. da Silva 232 & ER \\
\hline Opuntia brasiliensis (Briton \& Rose) P.J. Braun \& Esteves & A.G. da Silva 328 & $\mathrm{ARB}$ \\
\hline Rhipsalis cereuscula Haw. & C.A.M. Oliveira 25 & ER-E \\
\hline \multicolumn{3}{|l|}{ Campanulaceae } \\
\hline Centropogon cornutus (L.) Druce & C.A.M. Oliveira 17 & SUB \\
\hline \multicolumn{3}{|l|}{ Celastraceae (R.M.C. Okano - VIC) } \\
\hline Maytenus erythroxylon Reissek & L.M. Nascimento 299 & ARV \\
\hline M. patens Reissek & L.M. Nascimento 270 & ARV \\
\hline \multicolumn{3}{|l|}{ Chrysobalanaceae } \\
\hline Hirtella racemosa Lam. & L.M. Nascimento 144 & ARV \\
\hline \multicolumn{3}{|l|}{ Clusiaceae } \\
\hline Clusia hilariana Schltdl. & L.M. Nascimento 169 & ARV \\
\hline \multicolumn{3}{|l|}{ Combretaceae } \\
\hline Buchenavia capitata (Vahl) Eichler & L.M. Nascimento 320 & $\mathrm{ARV}$ \\
\hline \multicolumn{3}{|l|}{ Commelinaceae } \\
\hline Dichorisandra hexandra (Aubl.) Standl. & L.M. Nascimento 236 & ER \\
\hline J. martii Choisy & C.A.M. Oliveira 5 & $\mathrm{~T}$ \\
\hline Merremia macrocalyx (Ruiz \& Pav.) O’Donell. & L.M. Nascimento 461 & $\mathrm{~T}$ \\
\hline \multicolumn{3}{|l|}{ Convolvulaceae } \\
\hline Jacquemontia cf. bahiensis O'Donell. & A.G. da Silva 119 & $\mathrm{TR}$ \\
\hline \multicolumn{3}{|l|}{ Cunoniaceae (C. Zickel - PEUFR) } \\
\hline Lamanonia ternata Vell. & L.M. Nascimento 340 & ARV \\
\hline \multicolumn{3}{|l|}{ Dilleniaceae } \\
\hline Doliocarpus glomeratus Eichler & L.M. Nascimento 161 & $\mathrm{~T}$ \\
\hline \multicolumn{3}{|l|}{ Erythroxylaceae (M.I.B. Loiola - UFRN) } \\
\hline Erythroxylum blanchetii O E. Schulz & A.G. da Silva 12 & ARV \\
\hline E. citrifolium A. St.-Hil. & L.M. Nascimento 226 & ARV \\
\hline E. columbinum Mart. & L.M. Nascimento 237 & ARV \\
\hline E. flaccidum Salzm. ex Peyr. & A.M. da Silva 76 & ARV \\
\hline E. revolutum Mart. & L.M. Nascimento 216 & ARV \\
\hline \multicolumn{3}{|l|}{ Euphorbiaceae (M.F.Lucena - UFP; A. Laurênio - PEUFR) } \\
\hline Acalypha brasiliensis Müll. Arg. & A.M. da Silva 77 & SUB \\
\hline
\end{tabular}




\begin{tabular}{|c|c|c|}
\hline FAMÍLIA/ESPÉCIE & COLETOR/No & НÁBITO \\
\hline Actinostemon concolor (Spreng.) Müll. Arg. & A.G. da Silva 246 & $\mathrm{ARB}$ \\
\hline Bernardia tamanduana (Baill.) Müll. Arg. & L.M. Nascimento 113 & $\mathrm{ARB}$ \\
\hline Cnidoscolus halteris Fern.Casas & L.M. Nascimento 465 & ARB \\
\hline Croton floribundus Spreng. & L.M. Nascimento 316 & ARV \\
\hline C. heliotropiifolius Kunth & A.G. da Silva 217 & ARB \\
\hline C. pulegioides Baill. & A.G. da Silva 13 & SUB \\
\hline C. rhamnifolius Willd. & L.M. Nascimento 399 & ARB \\
\hline Sebastiania jacobinensis (Müll. Arg.) Müll. Arg. & A.G. da Silva 297 & ARV \\
\hline Sebastiania sp. & L.M. Nascimento 377 & ARV \\
\hline Sapium sp. & A.G. da Silva 361 & ARV \\
\hline Stilingia trapezoidea Ule & L.M. Nascimento 520 & $\mathrm{ARV}$ \\
\hline Tragia sellowiana (Baill.) Müll. Arg. & L.M. Nascimento 124 & $\mathrm{~T}$ \\
\hline \multicolumn{3}{|c|}{ Fabaceae (H.C. Lima - RB; F.C.P. Garcia - VIC; L.P. Queiroz - HUEFS) } \\
\hline Acacia glomerosa Benth. & A.G. da Silva 1 & ARV \\
\hline A. limae Bocage \& Miotto & A.G. da Silva 290 & ARV \\
\hline A. paniculata Willd. & L.M. Nascimento 360 & ARV \\
\hline Albizia polycephala (Benth.) Killip & L.M. Nascimento 499 & ARV \\
\hline Andira ormosioides Benth. & L.M. Nascimento 498 & ARV \\
\hline Bowdichia virgilioides Kunth & L.M. Nascimento 145 & ARV \\
\hline Calopogonium caeruleum (Benth.) C. Wright ex Salvalle & C.A.M. Oliveira 41 & $\mathrm{~T}$ \\
\hline Canavalia parviflora Benth. & A.G. da Silva 60 & $\mathrm{~T}$ \\
\hline Chamaecrista pascuorum (Mart. ex Benth.) H.S. Irwin \& Barneby & A.G. da Silva 317 & SUB \\
\hline Copaifera trapezifolia Hayne & L.M. Nascimento 506 & $\mathrm{ARV}$ \\
\hline Dalbergia cf. cearensis Ducke & L.M. Nascimento 481 & ARV \\
\hline Dioclea guianensis Benth. & A.G. da Silva 315 & $\mathrm{~T}$ \\
\hline Erythrina velutina Willd. & L.M. Nascimento 191 & ARV \\
\hline Enterolobium contortisiliquum (Vell.) Morong & L.M. Nascimento 112 & ARV \\
\hline Hymenolobium flavum Kleinhoonte & L.M. Nascimento 271 & ARV \\
\hline Inga marginata Willd. & L.M. Nascimento 109 & ARV \\
\hline I. striata Benth. & L.M. Nascimento 132 & ARV \\
\hline I. subnuda Salzm. ex Benth. subsp. subnuda & A.G.da Silva 159 & $\mathrm{ARV}$ \\
\hline I. vera Willd. subsp. affinis (DC.) T.D. Penn. & A.G. da Silva 306 & ARV \\
\hline Lonchocarpus cultratus (Vell.) A.M.G. Azevedo \& H.C. Lima & A.G. da Silva 227 & ARV \\
\hline Machaerium hirtum (Vell.) Stellfeld & L.M. Nascimento 411 & ARV \\
\hline Mimosa arenosa (Willd.) Poir. & A.G. da Silva 41 & $\mathrm{ARB}$ \\
\hline M. borboremae Harms & D.C. Silva 67 & ARV \\
\hline M. sensitiva $\mathrm{L}$. & L.M. Nascimento 260 & $\mathrm{ARB}$ \\
\hline Pterocarpus rohrii Vahl & L.M. Nascimento 351 & ARV \\
\hline
\end{tabular}




\begin{tabular}{|c|c|c|}
\hline FAMÍLIA/ESPÉCIE & COLETOR/No & HÁBITO \\
\hline Piptadenia stipulacea (Benth.) Ducke & A.G. da Silva 7 & ARV \\
\hline Senegalia piauhiensis (Benth.) Bocage \& L.P. Queiroz & A.G. da Silva 384 & ARV \\
\hline Senna lechriosperma H.S. Irwin \& Barneby & A.G. da Silva 415 & ARV \\
\hline S. macranthera (DC. ex Collad.) H.S. Irwin \& Barneby & A.G. da Silva 27 & ARV \\
\hline Stylosanthes scabra Vogel & L.M. Nascimento 334 & ER \\
\hline Zollernia ilicifolia (Brongn.) Vogel & L.M. Nascimento 197 & ARV \\
\hline \multicolumn{3}{|l|}{ Gentianaceae } \\
\hline Irlbachia purpurascens (Aubl.) Maas & A.G. da Silva 129 & ER \\
\hline \multicolumn{3}{|l|}{ Gesneriaceae } \\
\hline Paliavana tenuiflora Mansf. & L.M. Nascimento 397 & ARB \\
\hline \multicolumn{3}{|l|}{ Heliconiaceae } \\
\hline Heliconia psittacorum L. f. & L.M. Nascimento 437 & ER \\
\hline \multicolumn{3}{|l|}{ Lauraceae (R. Barreto - UFP; Henk van der Werff - MO) } \\
\hline Nectandra cuspidata Nees \& Mart. & L.M. Nascimento 154 & ARV \\
\hline Ocotea aff. elegans Mez & L.M. Nascimento 386 & ARV \\
\hline O. glomerata $(\mathrm{Ness}) \mathrm{Mez}$ & L.M. Nascimento 227 & ARV \\
\hline Ocotea sp. & L.M. Nascimento 429 & ARV \\
\hline \multicolumn{3}{|l|}{ Loasaceae } \\
\hline Loasa rupestris Gardner & L.M. Nascimento 247 & ER \\
\hline \multicolumn{3}{|l|}{ Loranthaceae } \\
\hline Struthanthus marginatus (Desr.) Blume & A.G. da Silva 11 & ER-H \\
\hline \multicolumn{3}{|l|}{ Lythraceae } \\
\hline Cuphea circaeoides Sm. ex Sims & L.M. Nascimento 435 & ER \\
\hline \multicolumn{3}{|l|}{ Malpighiaceae } \\
\hline Byrsonima crispa A. Juss. & L.M. Nascimento 212 & ARV \\
\hline B. cf. nitidifolia A. Juss. & A.M. da Silva 51 & ARV \\
\hline B. verbascifolia (L.) DC. & A.G. da Silva 239 & ARV \\
\hline Byrsonima sp.1 & L.M. Nascimento 352 & ARV \\
\hline Byrsonima sp.2 & L.M. Nascimento 477 & ARV \\
\hline Byrsonima sp.3 & L.M. Nascimento 138 & ARV \\
\hline Galphimia brasiliensis A. Juss. & L.M. Nascimento 249 & ER \\
\hline Tetrapteris cf. mucronata Cav. & C.A.M. Oliveira 24 & $\mathrm{~T}$ \\
\hline \multicolumn{3}{|l|}{ Malvaceae (G.S. Baracho - UFP; M.C. Tschá - PEUFR) } \\
\hline Bakeridesia pickelii Monteiro & A.G. da Silva 118 & $\mathrm{ARB}$ \\
\hline Eriotheca crenulaticalyx A. Robyns & L.M. Nascimento 195 & ARV \\
\hline Sida salzmannii Monterio & A.G. da Silva 37 & ARB \\
\hline Triumfetta semitriloba Jacq. & L.M. Nascimento 424 & ARB \\
\hline \multicolumn{3}{|l|}{ Marantaceae (K. Yoshida Arns - IPA) } \\
\hline Ctenanthe sp. & L.M. Nascimento 196 & ER \\
\hline
\end{tabular}




\begin{tabular}{l}
\hline FAMÍLIA/ESPÉCIE \\
\hline Stromanthe glabra Yosh.-Arns \\
Melastomataceae (R. Goldenberg - UPCB)
\end{tabular}

Clidemia debilis Crueg.

L.M. Nascimento 125

ARB

C. hirta (L.) D. Don

L.M. Nascimento 124

ARB

Conostegia sp.

A.G. da Silva 91

ER

Huberia sp.

L.M. Nascimento 321

ARV

Marcetia taxifolia (A. St.-Hil.) DC.

A.G. da Silva 131

ER

Miconia albicans (Sw.) Tiana

L.M. Nascimento 179

ARV

M. caudigera (Mart. \& Schr.) DC.

L.M. Nascimento 156

ARV

M. ciliata (Rich.) DC.

A.G. da Silva 59

ARV

M. rimalis Naudin

L.M. Nascimento 213

ARV

M. rubiginosa (Bonpl.) DC.

A.G. da Silva 241

ARV

M. stenostachya DC.

L.M. Nascimento 183

ARB

Pterolepsis sp.

Tibouchina heteromalla Cogn.

A.G. da Silva 309

ER

A.G. da Silva 199

ARB

Meliaceae (T.D. Pennington - MO)

Cabralea canjerana (Vell.) Mart.

L.M. Nascimento 267

ARV

Cedrela odorata $\mathrm{L}$.

L.M. Nascimento 159

ARV

\section{Moraceae}

Brosimum guianense (Aubl.) Huber

L.M. Nascimento 385

ARV

\section{Myrtaceae (G.M. Barroso - RB)}

Campomanesia dardanolimai Mattos \& D. Legrand.

L.M. Nascimento 444

ARV

Eugenia aff. acutata Miq.

L.M. Nascimento 305

ARV

E. candolleana DC.

L.M. Nascimento 482

ARV

E. cerasiflora Miq.

L.M. Nascimento 276

ARV

E. ligustrina (Sw.) Willd.

L.M. Nascimento 295

ARV

E. obtusifolia Cambess.

L.M. Nascimento 335

ARB

E. punicifolia (Kunth) DC.

A.G. da Silva 148

ARV

Eugenia sp.1

A.G. da Silva 246

ARV

Eugenia sp.2

L.M. Nascimento 380

ARV

Marlierea clausseniana (O. Berg) Kiaersk.

L.M. Nascimento 184

ARV

M. luschnatiana (O. Berg) D. Legrand

L.M. Nascimento 246

ARV

Myrcia amazonica DC.

L.M. Nascimento 177

ARV

M. bracteata (Rich.) DC.

L.M. Nascimento 139

ARV

M. fallax (Rich.) DC.

C.A.M. Oliveira 51

ARV

M. aff. magnoliifolia DC.

L.M. Nascimento 339

ARV

M. obtecta (O. Berg) Kiaersk.

L.M. Nascimento 134

ARV

M. sylvatica (G. Mey.) DC.

L.M. Nascimento 137

ARV

M. tomentosa (Aubl.) DC.

L.M. Nascimento 203

ARV 


\begin{tabular}{l}
\hline FAMÍLIA/ESPÉCIE \\
\hline Myrcia sp.1 \\
Myrcia sp.2 \\
Myrcia sp.3 \\
Myrcia sp.4 \\
Myrciaria sp. \\
Psidium guineense Sw. \\
Nyctaginaceae (A. Furlan - SJRP) \\
Guapira hirtusa (Choisy) Lundell \\
G. laxa (Netto) Furlan \\
G. nitida (Schmidt) Lundell \\
G. opposita (Vell.) Reitz
\end{tabular}

COLETOR/N ${ }^{\circ}$

A.G. da Silva 338

L.M. Nascimento 486

A.G. da Silva 208

L.M. Nascimento 493

L.M. Nascimento 410

A.G. da Silva 4

L.M. Nascimento 219

L.M. Nascimento 307

L.M. Nascimento 225

L.M. Nascimento 304

L.M. Nascimento 186

A.G. da Silva 263

A.G. da Silva 109

A.G. da Silva 165

A.G. da Silva 108

A.G. da Silva 188

A.G. da Silva 312

L.M. Nascimento 456

L.M. Nascimento 455

A.G. da Silva 31

L.M. Nascimento 454

L.M. Nascimento 414

A.M. da Silva 53

L.M. Nascimento 297

A.G. da Silva 220

L.M.Nascimento 358

L.M. Nascimento 228

A.M. da Silva 52

A.G. da Silva 256

L.M. Nascimento 413

L.M. Nascimento 239
HÁBITO

ARV

ARV

ARV

ARV

ARV

ARV

ARV

ARV

ARV

ARV

ARV

ARV

ER-E

ER-E

ER-E

ER-E

ER-E

ER-E

ER

ER-E

ER-E

ER-E

ER-E

ER-E

ER

ER

ER

$\mathrm{T}$

SUB

ARV

Picramniaceae (W.W. Thomas - NYBG)

Picramnia gardneri Planch.

ARV 


\section{FAMÍLIA/ESPÉCIE}

P. crenata (Vell.) Hassl.

Piperaceae

Peperomia tetraphylla (G. Forst.) Hook. \& Arn.

Piper arboreum Aubl.

P. arboreum Aubl. subsp arboreum

\section{Poaceae (R.P. Oliveira - HUEFS)}

Axonopus aureus P. Beauv.

Axonopus sp.

Brachiaria plantaginea (Link) Hitchc.

Eragrotis aff. neesii Tin.

Lasiacis ligulata Hitchc. \& Chase

Melinis repens (Willd.) Zizka

Paspalus sp.

Raddia angustifolia Soderstr. \& Zuloaga

Polygonaceae

Coccoloba mollis Casar.

\section{Primulaceae}

Myrsine guianensis (Aubl.) Kuntze

\section{Proteaceae}

Roupala paulensis Sleumer

Colubrina glandulosa Perkins

Rubiaceae (D. Zappi - RBG)

Amaioua guianensis Aubl.

Borreria verticillata (L.) G. Mey.

Chiococca alba (L.) Hitchc.

Coutarea hexandra (Jacq.) K. Schum.

Guettarda virburnoides Cham. \& Schltdl.

Palicourea crocea (Sw.) Roem \& Schult.

Posoqueria latifolia (Rudge) Roem. \& Schult.

Psychotria bahiensis DC.

P. carthagenensis Jacq.

P. hoffmannseggiana (Willd. ex Roem.\& Schult.) Müll. Arg.

P. schlechtendaliana Müll. Arg.

P. sessilis (Vell.) Müll. Arg.

Randia armata (Sw.) DC.

Rudgea jacobinensis Müll. Arg.

Tocoyena formosa (Cham. \& Schltdl.) K. Schum.

L.M. Nascimento 333
L.M. Nascimento 418

L.M. Nascimento 398

A.G. da Silva 227

ER-E

A.G. da Silva 58

ARB

L.M. Nascimento 442

L.M. Nascimento 345

A.G. da Silva 318

L.M. Nascimento 392

A.M. da Silva 13

L.M. Nascimento 394

A.G. da Silva 259

A.G. da Silva 292

L.M. Nascimento 158

L.M. Nascimento 152

ARV

ARV

ARV

L.M. Nascimento 338

ARV

L.M. Nascimento 391

ER

A.G. da Silva 77

ARB

A.G. da Silva 24

ARB

L.M. Nascimento 208

ARV

L.M. Nascimento 217

ARB

A.G. da Silva 98

ARV

L.M. Nascimento 235

ARB

L.M. Nascimento 222

ARV

A.G. da Silva 42

SUB

L.M. Nascimento 129

ARB

L.M. Nascimento 162

ARV

A.G. da Silva 298

ARV

A.G. da Silva 93

ARV

A.G. da Silva 94

ARV

\section{Rutaceae}




\section{FAMÍLIA/ESPÉCIE}

Zanthoxylum fagara (L.) Sarg.

Salicaceae

Banara brasiliensis (Schott) Benth.

Casearia sylvestris $\mathrm{Sw}$.

Prockia crucis P. Browne ex L.

\section{Santalaceae}

Phoradendron crassifolium (Pohl ex DC.) Eichler

P. piauhyanum Tel.

Phoradendron sp.

\section{Sapindaceae (M.S. Ferrucci - CTES)}

Allophylus edulis (A. St.-Hil., Cambess. \& A. Juss.) Radlk.

Cupania emarginata Cambess.

C. oblongifolia Mart.

C. revoluta Rolfe

Dodonea viscosa (L.) Jacq.

Matayba mollis Radlk.

Paullinia trigonia Vell.

Serjania ichthyocotna Radlk.

Sapotaceae (T.D. Pennington - MO)

Chrysophyllum gonocarpum (Mart. \& Eichler ex Miq.) Engl.

Manilkara rufula (Miq.) H.J. Lam.

\section{Scrophulariaceae}

Angelonia salicariaefolia Humb. \& Bonpl.

Scoparia dulcis L.

Simaroubaceae (W.W. Thomas - NYBG)

Simarouba amara Aubl.

\section{Smilacaceae}

Smilax spruceana A. DC.

S. syphilitica Griseb.

Solanaceae (M. Agra - JPB; S. Knapp - NHM; M. Nee - NYBG)

Acnistus arborescens (L.) Schltdl.

L.M. Nascimento 317

L.M. Nascimento 165

L.M. Nascimento 218

L.M. Nascimento 232

L.M. Nascimento 107

A.G. da Silva 127

A.G. da Silva 170

L.M. Nascimento 491

L.M. Nascimento 314
HÁBITO

ARV

ARV

ARV

ARB

ER-H

ER-H

ER-H

ARV

ARV

ARV

ARV

ARB

ARV

$\mathrm{T}$

$\mathrm{T}$

ARV

ARV

SUB

ER

ARV

T

T

ARV

ARV

ARV

ARV

ARV

ARB

ARB

ARB

ARV 


\begin{tabular}{|c|c|c|}
\hline FAMÍLIA/ESPÉCIE & COLETOR/N ${ }^{\circ}$ & HÁBITO \\
\hline S. gemellum Sendtn. & L.M. Nascimento 406 & ARV \\
\hline S. paniculatum L. & C.A.M. Oliveira 36 & $\mathrm{ARB}$ \\
\hline S. paraibanum Agra & C.A.M. Oliveira 23 & ARB \\
\hline S. rhytidoandrum Sendtn. & A.G. da Silva 389 & $\mathrm{ARB}$ \\
\hline S. stipulaceum Roem. \& Schult. & L.M. Nascimento 221 & $\mathrm{ARB}$ \\
\hline S. swartzianum Roem. \& Schult. & L.M. Nascimento 409 & ARV \\
\hline \multicolumn{3}{|l|}{ Styracaceae } \\
\hline Styrax camporum Pohl & L.M. Nascimento 163 & ARV \\
\hline \multicolumn{3}{|l|}{ Symplocaceae } \\
\hline Symplocos nitens (Pohl) Benth. & L.M. Nascimento $511 \mathrm{~A}$ & ARV \\
\hline \multicolumn{3}{|l|}{ Ulmaceae } \\
\hline Trema micrantha $(\mathrm{L}$.$) Blume$ & L.M. Nascimento 264 & ARV \\
\hline \multicolumn{3}{|l|}{ Urticaceae } \\
\hline Urera baccifera (L.) Gaudich. ex Wedd. & C.A.M. Oliveira 22 & ARV \\
\hline \multicolumn{3}{|l|}{ Verbenaceae (F. França - HUEFS) } \\
\hline Aegiphila integrifolia (Jacq.) B.D. Jacks. & A.G. da Silva 248 & ARV \\
\hline A. luschnatii Schauer & A.G. da Silva 134 & $\mathrm{ARB}$ \\
\hline A. vitelliniflora Klotzsch ex Walp. & L.M. Nascimento 263 & $\mathrm{ARB}$ \\
\hline Lantana camara $\mathrm{L}$. & C.A.M. Oliveira 57 & $\mathrm{ARB}$ \\
\hline L. canescens Kunth & C.A.M. Oliveira 58 & $\mathrm{ARB}$ \\
\hline Vitex rufescens A. Juss. & L.M. Nascimento 513 & ARV \\
\hline \multicolumn{3}{|l|}{ Vochysiaceae } \\
\hline Vochysia thyrsoidea Pohl. & L.M. Nascimento 198 & ARV \\
\hline
\end{tabular}

